\title{
Conserved quantity of elastic waves in multi-layered media: 2D SH case - Normalized Energy Density
}

\section{$\operatorname{AUTHOR}(\mathrm{S})$ :}

Goto, Hiroyuki; Sawada, Sumio; Hirai, Toshiyuki

\section{CITATION:}

Goto, Hiroyuki ... [et al]. Conserved quantity of elastic waves in multi-layered media: 2D SH case - Normalized Energy Density. Wave Motion 2011, 48(7): 603-613

\section{ISSUE DATE:}

2011-11

URL:

http://hdl.handle.net/2433/147254

\section{RIGHT:}

(C) 2011 Elsevier B.V.; This is not the published version. Please cite only the published version.; この論文は出版社版でありません。引用の際に は出版社版をご確認ご利用ください。 


\title{
Conserved quantity of elastic waves in multi-layered media: 2D SH case -Normalized Energy Density-
}

\author{
Hiroyuki Goto $^{\mathrm{a}, *}$, Sumio Sawada ${ }^{\mathrm{a}}$, Toshiyuki Hirai ${ }^{\mathrm{b}}$ \\ ${ }^{a}$ Disaster Prevention Research Institute, Kyoto University, Gokasho, Uji, Kyoto \\ 611-0011, Japan \\ ${ }^{b}$ NEWJEC Inc., 2-3-20 Honjo-Higashi, Kita-ku, Osaka 531-0074, Japan
}

\begin{abstract}
We introduce a new conserved quantity, Normalized Energy Density (NED), alternative to the conventional definition of energy for a layered structure in a $2 \mathrm{D} \mathrm{SH}$ problem. NED is defined by the average of power of a half transfer function multiplied by the impedance, and the conservation across the material interface is analytically proved for a two-layered case. For three, four, and ten-layered cases, the conservation is examined by applying the Monte Carlo simulation method, and then NED is supposed to be conserved through the layers.
\end{abstract}

Keywords: Elastic wave, Energy

\section{Introduction}

Conserved quantities, such as mass, momentum and energy, in elastodynamic problems are the fundamental variables when analyzing wave propagation in a continuous medium. In addition, the balance principles associated with these quantities, e.g., the balance of mass and the balance of momentum, govern the deformation within the framework of Newtonian mechanics. The balance of energy is one of the principles used to quantify the seismic energy radiated from an earthquake source.

\footnotetext{
*Corresponding author. Tel.:+81 77438 4067; fax:+81 774384070.

Email address: goto@catfish.dpri.kyoto-u.ac.jp (Hiroyuki Goto)
} 
Radiation energy $E$ is theoretically defined as the total energy transmitted through a certain surface, $S$, as follows:

$$
E=-\int_{0}^{\infty} d t \int_{S}\left(\sigma_{i j}-\sigma_{i j}^{0}\right) \dot{u}_{i} n_{j} d S
$$

where $\sigma_{i j}$ and $\sigma_{i j}^{0}$ are the tentative and the initial stress tensors, respectively. $\dot{u}_{i}$ is the particle velocity, and $n_{j}$ is a normal vector of the surface $S$. When a particular region, e.g. a seismic fault, generates all of the energy, the integration on the arbitrary surface $S$ surrounding the region is theoretically conserved even for a general heterogeneous medium. The above representation has already been introduced in Love [1] . The energy of seismic events was first applied by Richter [2] in order to measure the size of earthquakes by using the local magnitude scale $\left(M_{L}\right)$, although it was not exactly equal to the definition of the energy. Afterward, Kanamori [3] proposed the use of moment magnitude $\left(M_{W}\right)$, defined from the seismic moment that is related to the energy release during the events, whose energy is different from the radiation energy (Equation (1)). A detailed discussion on radiation energy is introduced in Kostrov and Das [4], Fukuyama [5], and Abercrombie et al. [6].

If a seismic wave through the surface $S$ is approximated by a single plane wave, either a $\mathrm{P}$ - or an $\mathrm{S}$-wave propagated in a uniform direction, the energy for the P-wave case, $E_{\alpha}$, and that for the S-wave case, $E_{\beta}$, are represented as follows:

$$
E_{\alpha}=\int_{0}^{\infty} d t \int_{S} \rho \alpha \dot{u}_{\alpha}^{2} l_{i} n_{i} d S, \quad E_{\beta}=\int_{0}^{\infty} d t \int_{S} \rho \beta \dot{u}_{\beta}^{2} l_{i} n_{i} d S,
$$

where $\rho, \alpha$ and $\beta$ are the density, the $\mathrm{P}$-wave velocity, and the $\mathrm{S}$-wave velocity, respectively. $\dot{u}_{\alpha}$ and $\dot{u}_{\beta}$ are the amplitudes of particle velocity for the P-wave and the S-wave, respectively. $l_{i}$ is a vector representing the direction of the wave propagation. The energy density, defined by the integrand, is a product of the square of the particle velocity and the impedance. Note that the total energy for a general wave field, represented by the superposition of the Pand the S-waves, is not equal to $E_{\alpha}+E_{\beta}$ (see Appendix A).

A part of the energy integrated on the shrunken area of $S$ is utilized as a principle of energy conservation when all of the input energy is confined in a certain region, so-called "ray tube" [7]. The energy on the cross-sectional area of the tube is theoretically conserved. Here, we focus on the layered 


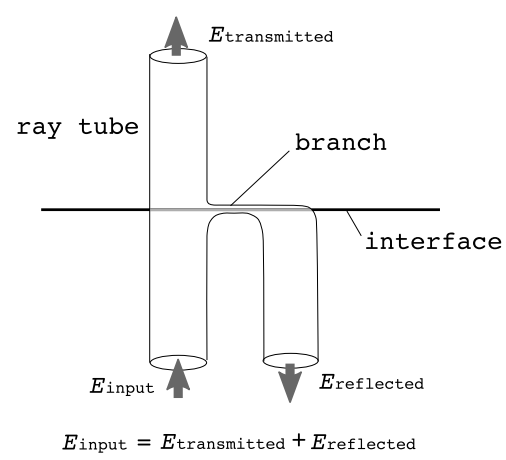

Figure 1: Ray tube at the material interface and the energy conservation.

structure. At the interface, part of the energy for the input wave is transmitted, and the rest is reflected. Then, both the transmitted and the reflected waves should be considered in order to apply the energy conservation in the ray tube. As shown in Figure 1, the sum of the transmitted energy and the reflected energy is equal to the input energy. However, the total input energy can not be observed in only the opposite layer because the transmitted energy is part of the input energy. Therefore, the energy is not conserved across the interfaces. Note that some researchers apply the energy, directly defined by $\int_{0}^{\infty} \rho c \dot{u}^{2} d t$, to the layered structure (e.g., Kokusho and Motoyama [8]), however, they do not pay attention to the fact that the quantity is not conserved. If a quantity conserved over the layer structure exists, absorbed energy in propagating in the layer might be estimated, directly. The quantification of the absorbed energy helps to understand the hysteretic damping due to anelasticity, e.g. Q-factor, and the soil nonlinearity, as discussed in Kokusho and Motoyama [8].

In this article, we introduce a quantity, Normalized Energy Density, which is an alternative to the conventional definition of energy, and discuss the features of the 2D SH problem. The quantity is analytically discussed for the two-layered case, and numerically examined for multi-layered cases.

\section{Two-layered case}

The theoretical implementation starts from the waves, vertically propagated into a simple two-layered structure. Only 2D SH waves, which have an antiplane amplitude with respect to the plane, are considered here. The structure consists of a horizontal layer, Layer \#1, with a thickness of $h$ and 


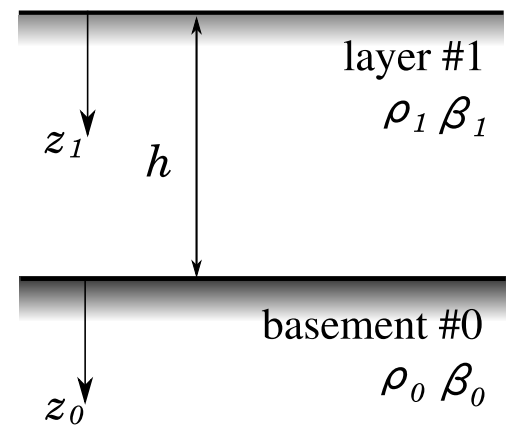

Figure 2: Two-layered model.

a half space basement, Basement \#0. The S-wave velocity and the density are $\beta_{1}$ and $\rho_{1}$ for Layer $\# 1$ and $\beta_{0}$ and $\rho_{0}$ for Basement \#0, as shown in Figure 2. An incident plane wave propagates vertically into Layer \#1 through the interface between Layer \#1 and Basement \#0. Each layer keeps elasticity independent of the wave amplitude.

The displacement in each layer satisfies the following wave equations in the frequency domain:

$$
\begin{aligned}
-\frac{\omega^{2}}{\beta_{1}^{2}} u_{1}\left(\omega, z_{1}\right) & =\frac{\partial^{2} u_{1}\left(\omega, z_{1}\right)}{\partial z_{1}^{2}} \\
-\frac{\omega^{2}}{\beta_{0}^{2}} u_{0}\left(\omega, z_{0}\right) & =\frac{\partial^{2} u_{0}\left(\omega, z_{0}\right)}{\partial z_{0}^{2}}
\end{aligned}
$$

where $\omega$ is an angular frequency, and $u_{1}$ and $u_{0}$ are the displacements of Layer \#1 and Basement \#0, respectively. The general solutions for Equations (3) and (4) are represented by the superposition of upgoing and downgoing waves, namely,

$$
\begin{aligned}
& u_{1}\left(\omega, z_{1}\right)=A_{1} e^{i \omega z_{1} / \beta_{1}}+B_{1} e^{-i \omega z_{1} / \beta_{1}} \\
& u_{0}\left(\omega, z_{0}\right)=A_{0} e^{i \omega z_{0} / \beta_{0}}+B_{0} e^{-i \omega z_{0} / \beta_{0}}
\end{aligned}
$$

where $i$ indicates an imaginary unit. $A_{1}$ and $A_{0}$ are the amplitudes of the upgoing waves, while $B_{1}$ and $B_{0}$ are those for the downgoing waves. The boundary conditions at the interface are as follows:

$$
u_{1}(\omega, h)=u_{0}(\omega, 0),\left.\quad \rho_{1} \beta_{1}^{2} \frac{\partial u_{1}}{\partial z_{1}}\right|_{z_{1}=h}=\left.\rho_{0} \beta_{0}^{2} \frac{\partial u_{0}}{\partial z_{0}}\right|_{z_{0}=0} .
$$


Then, the amplitudes for Basement \#0 are represented by those for Layer \#1 via the following matrix equation.

$$
\left(\begin{array}{c}
A_{0} \\
B_{0}
\end{array}\right)=\left[\begin{array}{ll}
\frac{1}{2}\left(1+R_{1,0}\right) e^{i \omega h / \beta_{1}}, & \frac{1}{2}\left(1-R_{1,0}\right) e^{-i \omega h / \beta_{1}} \\
\frac{1}{2}\left(1-R_{1,0}\right) e^{i \omega h / \beta_{1}}, & \frac{1}{2}\left(1+R_{1,0}\right) e^{-i \omega h / \beta_{1}}
\end{array}\right]\left(\begin{array}{c}
A_{1} \\
B_{1}
\end{array}\right)
$$

where $R_{1,0}$ represents an impedance ratio $\left(=\rho_{1} \beta_{1} / \rho_{0} \beta_{0}\right)$. Since the space differentiation of the displacement is zero on the free surface, the amplitude of an upgoing wave, $A_{1}$, should be equal to that of a downgoing wave, $B_{1}$. Then, the ratio, $A_{1} / A_{0}$, is represented by the following form:

$$
\frac{A_{1}}{A_{0}}=\frac{1}{\cos \left(\omega h / \beta_{1}\right)+i R_{1,0} \sin \left(\omega h / \beta_{1}\right)} .
$$

Quantity $A_{1} / A_{0}$ is half of transfer function $H(\omega)$, ratio of free surface displacement to input wave, for the two-layered structure,

$$
H(\omega) \equiv \frac{u_{1}(\omega, 0)}{A_{0}}=\frac{2 A_{1}}{A_{0}} .
$$

Here, we define real functions $P_{1}$ and $P_{0}$ as the square of the absolute value of the upgoing wave amplitudes $A_{1}$ and $A_{0}$ normalized by input wave amplitude.

$$
\begin{aligned}
& P_{1}(\omega)=\left|\frac{A_{1}}{A_{0}}\right|^{2}=\frac{1}{\cos ^{2}\left(\omega h / \beta_{1}\right)+R_{1,0}{ }^{2} \sin ^{2}\left(\omega h / \beta_{1}\right)}, \\
& P_{0}(\omega)=\left|\frac{A_{0}}{A_{0}}\right|^{2}=1 .
\end{aligned}
$$

$P_{1}$ is a single-valued function with respect to $\cos \left(\omega h / \beta_{1}\right)$, and a periodic function of $\omega h / \beta_{1}=n \pi(n \in \mathbb{N})$. Moreover, $P_{1}$, defined in $\omega h / \beta_{1} \in[0, \pi]$, is symmetric about $\pi / 2$. Therefore, the average of $P$ in $\omega \in[-\infty, \infty]$, defined by $\left\langle P_{1}\right\rangle$, is equal to the average of $P_{1}$ in $\omega h / \beta_{1} \in[0, \pi / 2]$.

$$
\begin{aligned}
\left\langle P_{1}\right\rangle & =\lim _{\Omega \rightarrow \infty} \frac{1}{\Omega} \int_{-\Omega / 2}^{\Omega / 2} \frac{1}{\cos ^{2}\left(\omega h / \beta_{1}\right)+R_{1,0}{ }^{2} \sin ^{2}\left(\omega h / \beta_{1}\right)} d \omega \\
& =\frac{2}{\pi} \int_{0}^{\pi / 2} \frac{d \tilde{\omega}}{\cos ^{2}(\tilde{\omega})+R_{1,0}^{2} \sin ^{2}(\tilde{\omega})},
\end{aligned}
$$


where $\tilde{\omega}=\omega h / \beta_{1}$. The above integration is analytically integrable, as follows:

$$
\int_{0}^{\pi / 2} \frac{d \tilde{\omega}}{\cos ^{2}(\tilde{\omega})+R_{1,0}^{2} \sin ^{2}(\tilde{\omega})}=\left[\frac{1}{R_{1,0}} \tan ^{-1}\left(R_{1,0} \tan (\tilde{\omega})\right)\right]_{0}^{\pi / 2}=\frac{\pi}{2 R_{1,0}},
$$

Thus, the average of $P_{1}$ is equal to the inverse of impedance ratio $R_{1,0}$.

$$
\left\langle P_{1}\right\rangle=\frac{1}{R_{1,0}}=\frac{\rho_{0} \beta_{0}}{\rho_{1} \beta_{1}} .
$$

When the input wave satisfies $\left|A_{0}\right|=1,\left\langle P_{1}\right\rangle$ represents the average power for the upgoing waves in Layer \# 1 or for half the amplitude of the waves observed on the free surface. On the other hand, the average of $P_{0}$ is identical to 1 because of $P_{0}=1$.

$$
\left\langle P_{0}\right\rangle=1 \text {. }
$$

We define a quantity, a product of the average of $P$ and the impedance $\rho \beta$, such as $\rho_{1} \beta_{1}\left\langle P_{1}\right\rangle$ for Layer $\# 1$ and $\rho_{0} \beta_{0}\left\langle P_{0}\right\rangle$ for Basement $\# 0$. From the explicit representations of $\left\langle P_{1}\right\rangle$ and $\left\langle P_{0}\right\rangle$ by Eqs.(15)-(16), the following relation is obtained:

$$
\rho_{1} \beta_{1}\left\langle P_{1}\right\rangle=\rho_{0} \beta_{0}\left\langle P_{0}\right\rangle
$$

Equation (17) includes some physical features. Both the left- and righthand sides are the average power of the upgoing waves multiplied by the impedance at each layer. This means that the quantity, $\rho \beta\langle P\rangle$, is conserved across the interface. Moreover, the quantity is directly evaluated from the transfer function $H(\omega)$ as

$$
\rho_{1} \beta_{1}\left\langle P_{1}\right\rangle=\lim _{\Omega \rightarrow \infty} \frac{1}{\Omega} \int_{-\Omega / 2}^{\Omega / 2} \rho_{1} \beta_{1}\left|\frac{H(\omega)}{2}\right|^{2} d \omega .
$$

Let $g(t)$ to be an impulse response in a time domain for the upgoing waves in Layer \#1. Although $g(t)$ is not guaranteed to be a stationary ergodic process, the formally defined average power spectral density is generally equal to $\left\langle P_{1}\right\rangle$. Since the average power spectral density for a stationary ergodic process is a variance of the process, the quantity may be formally defined as a product of the impedance $\rho_{1} \beta_{1}$ and the variance of $g(t)$, as follows:

$$
\rho_{1} \beta_{1}\left\langle P_{1}\right\rangle=\lim _{T \rightarrow \infty} \frac{1}{T} \int_{-T / 2}^{T / 2} \rho_{1} \beta_{1}[g(t)-\langle g(t)\rangle]^{2} d t
$$




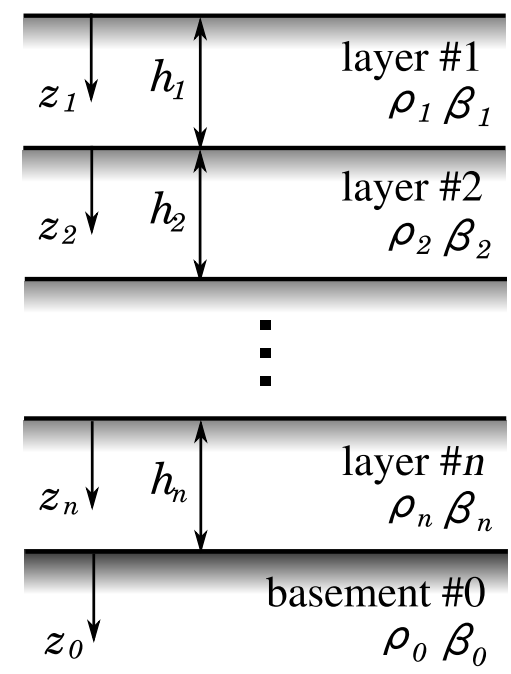

Figure 3: Multi-layered model.

where $\langle g(t)\rangle$ is the average $g(t)$. Note that the derivation of Equation (19) should be discussed after the treatment for the convergence of the integration, the super function, etc. We just mention that the representation of Equation (19) is similar to the conventional definition of the energy, whereas the proposed quantity, which is limited to the unity input waves, gives rise to conservation across the interface. Therefore, we name the quantity $\rho \beta\langle P\rangle$, Normalized Energy Density (NED).

\section{Multi-layered case}

We consider a multi-layered structure consisting of $n$ layers $(\# 1-\# n)$ over Basement \#0, as shown in Figure 3. The S-wave velocity of Layer \#k is $\beta_{k}$, the density is $\rho_{k}$, and the thickness is $h_{k}$. 2D SH waves vertically propagate vertically into the layers through the interface between Layer $\# n$ and Basement \#0.

The general solution for the wave equation at Layer $\# k$ is obtained by

$$
u_{k}\left(\omega, z_{k}\right)=A_{k} e^{i \omega z_{k} / \beta_{k}}+B_{k} e^{-i \omega z_{k} / \beta_{k}} .
$$

From the boundary conditions at the interface between Layers $\#(k+1)$ and $\# k$, the amplitudes for Layer $\#(k+1)$ are represented by those for Layer 
$\# \mathrm{k}$ as

$$
\left(\begin{array}{c}
A_{k+1} \\
B_{k+1}
\end{array}\right)=\left[\begin{array}{ll}
\frac{1}{2}\left(1+R_{k, k+1}\right) e^{i \omega h_{k} / \beta_{k}}, & \frac{1}{2}\left(1-R_{k, k+1}\right) e^{-i \omega h_{k} / \beta_{k}} \\
\frac{1}{2}\left(1-R_{k, k+1}\right) e^{i \omega h_{k} / \beta_{k}}, & \frac{1}{2}\left(1+R_{k, k+1}\right) e^{-i \omega h_{k} / \beta_{k}}
\end{array}\right]\left(\begin{array}{c}
A_{k} \\
B_{k}
\end{array}\right)
$$

where $A_{k}$ and $A_{k+1}$ are the amplitudes for the upgoing waves, and $B_{k}$ and $B_{k+1}$ are those for the downgoing waves. $R_{k, k+1}$ is the impedance ratio between Layers $\# k$ and $\#(k+1)\left(=\rho_{k} \beta_{k} / \rho_{k+1} \beta_{k+1}\right)$. Hereinafter, each component of the matrix in Equation (21) is indicated by $T_{i j}^{k}$ as

$$
\left[\begin{array}{ll}
T_{11}^{k} & T_{12}^{k} \\
T_{21}^{k} & T_{22}^{k}
\end{array}\right]=\left[\begin{array}{ll}
\frac{1}{2}\left(1+R_{k, k+1}\right) e^{i \omega h_{k} / \beta_{k}}, & \frac{1}{2}\left(1-R_{k, k+1}\right) e^{-i \omega h_{k} / \beta_{k}} \\
\frac{1}{2}\left(1-R_{k, k+1}\right) e^{i \omega h_{k} / \beta_{k}}, & \frac{1}{2}\left(1+R_{k, k+1}\right) e^{-i \omega h_{k} / \beta_{k}}
\end{array}\right],
$$

The amplitudes for Layer \# $k$ are represented by those for Layer \#1 by applying Equation (21), recursively.

$$
\begin{gathered}
\left\{\begin{array}{l}
A_{k}=T_{1 i}^{k-1} \cdot T_{i j}^{k-2} \cdots T_{l m}^{2} \cdot\left(T_{m 1}^{1} A_{1}+T_{m 2}^{1} B_{1}\right) \\
B_{k}=T_{2 i}^{k-1} \cdot T_{i j}^{k-2} \cdots T_{l m}^{2} \cdot\left(T_{m 1}^{1} A_{1}+T_{m 2}^{1} B_{1}\right)
\end{array} \quad(\text { for } k \geq 3)\right. \\
\left\{\begin{array}{l}
A_{2}=T_{11}^{1} A_{1}+T_{12}^{1} B_{1} \\
B_{2}=T_{21}^{1} A_{1}+T_{22}^{1} B_{1}
\end{array}\right.
\end{gathered}
$$

The traction-free condition on the free surface, $A_{1}=B_{1}$, gives the following representation of the amplitudes:

$$
\begin{gathered}
\left\{\begin{array}{l}
A_{k}=C_{k-1} A_{1} \\
B_{k}=D_{k-1} A_{1}
\end{array} \quad(\text { for } 1 \leq k \leq n)\right. \\
\left\{\begin{array}{l}
A_{0}=C_{n} A_{1} \\
B_{0}=D_{n} A_{1}
\end{array}\right.
\end{gathered}
$$

where $C_{k}$ and $D_{K}$ are defined as follows:

$$
\begin{gathered}
\left\{\begin{array}{l}
C_{k-1}=T_{1 i}^{k-1} \cdot T_{i j}^{k-2} \cdots T_{l m}^{2} \cdot\left(T_{m 1}^{1}+T_{m 2}^{1}\right) \\
D_{k-1}=T_{2 i}^{k-1} \cdot T_{i j}^{k-2} \cdots T_{l m}^{2} \cdot\left(T_{m 1}^{1}+T_{m 2}^{1}\right)
\end{array} \quad(\text { for } k \geq 2)\right. \\
\left\{\begin{array}{l}
C_{1}=T_{11}^{1}+T_{12}^{1} \\
D_{1}=T_{21}^{1}+T_{22}^{1}
\end{array}, \quad\left\{\begin{array}{l}
C_{0}=1 \\
D_{0}=1
\end{array}\right.\right.
\end{gathered}
$$


We introduce the identity of $C_{k}^{*}=D_{k}$ in the Appendix $\mathrm{B}$, where $C_{k}^{*}$ is a complex conjugate of $C_{k}$.

$P_{k}(\omega)$ is also defined by the square of the absolute value of $A_{k} / A_{0}$, as follows:

$$
P_{k}(\omega)=\left|\frac{A_{k}}{A_{0}}\right|^{2}=\frac{\left|C_{k-1}\right|^{2}}{\left|C_{n}\right|^{2}}
$$

The denominator of the last part of Equation (29) is rewritten as

$$
\begin{aligned}
\left|C_{n}\right|^{2} & =\left|T_{11}^{n} C_{n-1}+T_{12}^{n} D_{n-1}\right|^{2} \\
& =\left|\frac{1}{2}\left(1+R_{n, 0}\right) \alpha_{n} C_{n-1}+\frac{1}{2}\left(1-R_{n, 0}\right) \alpha_{n}^{*} C_{n-1}^{*}\right|^{2} \\
& =\left|\Re\left[\alpha_{n} C_{n-1}\right]+i R_{n, 0} \Im\left[\alpha_{n} C_{n-1}\right]\right|^{2} \\
& =\left(\Re\left[\alpha_{n} C_{n-1}\right]\right)^{2}+R_{n, 0}^{2}\left(\Im\left[\alpha_{n} C_{n-1}\right]\right)^{2},
\end{aligned}
$$

where $\alpha_{n}$ represents the complex variables defined by $\alpha_{n}=e^{i \omega h_{n} / \beta_{n}} . \Re[]$ and $\Im[]$ indicate the real and the imaginary parts of the argument, respectively. Therefore, the average of $P_{1},\left\langle P_{1}\right\rangle$, is represented as follows:

$$
\left\langle P_{1}\right\rangle=\lim _{\Omega \rightarrow \infty} \frac{1}{\Omega} \int_{-\Omega / 2}^{\Omega / 2} \frac{d \omega}{\left(\Re\left[\alpha_{n} C_{n-1}\right]\right)^{2}+R_{n, 0}^{2}\left(\Im\left[\alpha_{n} C_{n-1}\right]\right)^{2}} .
$$

For the two-layered case $(n=1)$, the above equation becomes Equation (13).

For the three-layered case $(n=2), \alpha_{2} C_{1}$ is a sum of periodic functions of $\omega\left(h_{1} / \beta_{1}+h_{2} / \beta_{2}\right)=m \pi$ and $\omega\left(h_{1} / \beta_{1}-h_{2} / \beta_{2}\right)=n \pi(m, n \in \mathbb{N})$, as follows:

$$
\alpha_{2} C_{1}=\frac{1}{2}\left(1+R_{1,2}\right) e^{i \omega\left(h_{1} / \beta_{1}+h_{2} / \beta_{2}\right)}+\frac{1}{2}\left(1-R_{1,2}\right) e^{-i \omega\left(h_{1} / \beta_{1}-h_{2} / \beta_{2}\right)}
$$

If the ratio $\left(h_{1} / \beta_{1}+h_{2} / \beta_{2}\right) /\left(h_{1} / \beta_{1}-h_{2} / \beta_{2}\right)$ were a rational number represented by $m / n(m, n \in \mathbb{N}), \alpha_{2} C_{1}$ might be a periodic function of $\omega\left(h_{1} / \beta_{1}+\right.$ $\left.h_{2} / \beta_{2}\right)=\ln \pi(l \in \mathbb{N})$. However, $\alpha_{2} C_{1}$ is generally not guaranteed to be a periodic function because $\left(h_{1} / \beta_{1}+h_{2} / \beta_{2}\right) /\left(h_{1} / \beta_{1}-h_{2} / \beta_{2}\right)$ is a real number. Therefore, the same strategy with the two-layered case (Eq.(13)) is not applicable to cases with more than three layers $(n \geq 2)$.

If the integration results become $1 / R_{1,0}, \mathrm{NED} \rho_{1} \beta_{1}\left\langle P_{1}\right\rangle$ is also equal to $\rho_{0} \beta_{0}\left\langle P_{0}\right\rangle$. In the same way, the average power of the upgoing waves in Layer $\# k(k \geq 2)$ is obtained as follows:

$$
\left\langle P_{k}\right\rangle=\lim _{\Omega \rightarrow \infty} \frac{1}{\Omega} \int_{-\Omega / 2}^{\Omega / 2} \frac{\left(\Re\left[\alpha_{k-1} C_{k-2}\right]\right)^{2}+R_{k-1, k}^{2}\left(\Im\left[\alpha_{k-1} C_{k-2}\right]\right)^{2}}{\left(\Re\left[\alpha_{n} C_{n-1}\right]\right)^{2}+R_{n, 0}^{2}\left(\Im\left[\alpha_{n} C_{n-1}\right]\right)^{2}} d \omega .
$$




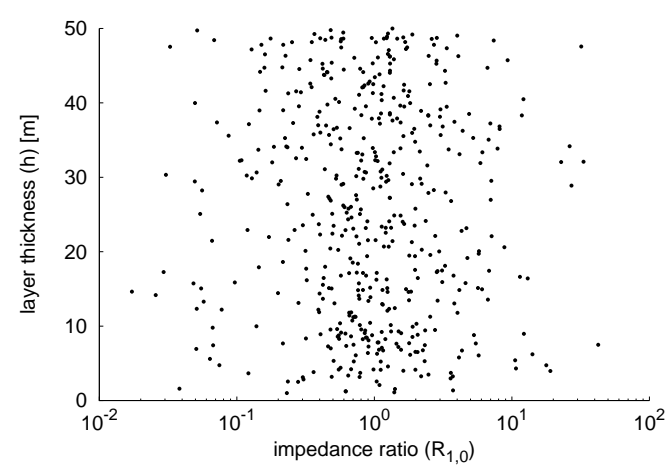

Figure 4: Parameter distribution of the impedance ratio and the layer thickness for the two-layered case.

If the integration results becomes $1 / R_{k, 0}$, NED is conserved through all the layers. Therefore, we apply the Monte Carlo simulation method in order to examine the conservation of NED for the cases with more than three layers in the latter chapter.

The above discussion is based on the vertical incident of SH waves. In the shallow layers of the crust structure within about 0-1000 $\mathrm{m}$ depth, the incident of waves from the basement is assumed to be vertical. On the other hand, the general oblique incidence of waves is also interesting. When the incident angle $\delta$ and the S-wave velocity $\beta_{k}$ satisfy $\beta_{0}>\beta_{k} \sin \delta$ in all layers, the problems are reduced to the same with the vertical incidence (see Appendix C).

\section{Numerical tests}

Firstly, the conservation of the quantity is numerically verified for the two-layered case using the Monte Carlo simulation. Five hundred sets of physical values are generated from random numbers within the range of 10$700 \mathrm{~m} / \mathrm{s}$ for the S-wave velocity and $1000-2000 \mathrm{~kg} / \mathrm{m}^{3}$ for the density of both Layer \#1 and Basement \#0. The parameters set for Layer \#1 are not guaranteed to be smaller than those for Basement \#0 in the simulations. The layer thickness is generated within the range of 1-50 m. Figure 4 shows the parameter distribution of impedance ratio $R_{1,0}$ and layer thickness $h$.

For each sample, $P_{1}(\omega)$ is calculated by Equation (11). Figure 5 shows three samples of $P_{1}(\omega)$ normalized by $1 / R_{1,0}$. Since the average $P_{1}(\omega)$ is 


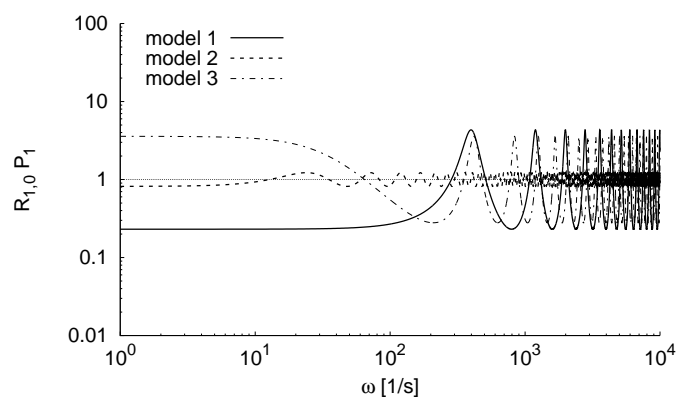

Figure 5: Samples of $P_{1}$ normalized by $1 / R_{1,0}$ for the two-layered case (Model1: $R_{1,0}=0.231, h / \beta_{1}=0.004 \mathrm{~s}$, Model2: $R_{1,0}=0.816, h / \beta_{1}=0.066 \mathrm{~s}$, Model3: $R_{1,0}=0.145$, $\left.h / \beta_{1}=0.249 \mathrm{~s}\right)$.

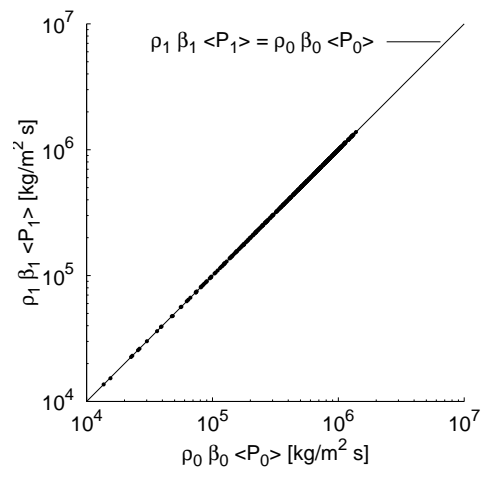

Figure 6: Comparison of NED between Layer \#1 and Basement \#0 for the two-layered case.

$1 / R_{1,0}$, as proved in section 2 , the average $R_{1,0} P_{1}(\omega)$ should be 1 . The amplitudes and the periodicity of $R_{1,0} P_{1}(\omega)$ are varied among the three samples, Model 1, 2, and 3, although the values are distributed around 1.

In order to check the conservation of NED the averaged values for $\rho_{1} \beta_{1} P_{1}$ are compared to those for $\rho_{0} \beta_{0} P_{0}$. The integrations are approximated by a numerical integration every $1.0 \mathrm{~s}^{-1}$ within $1.0 \mathrm{~s}^{-1} \leq \omega \leq 2.5 \times 10^{5} \mathrm{~s}^{-1}$. Figure 6 shows a comparison of NED. A line for $\rho_{1} \beta_{1}\left\langle P_{1}\right\rangle=\rho_{0} \beta_{0}\left\langle P_{0}\right\rangle$ is plotted together. The simulated samples, indicated by the black points, are exactly on the line, and thus, the conservation of NED is verified for the two-layered case.

For the three- and the four-layered cases, Monte Carlo simulations are also performed. Five hundred sets of physical values are also generated from 


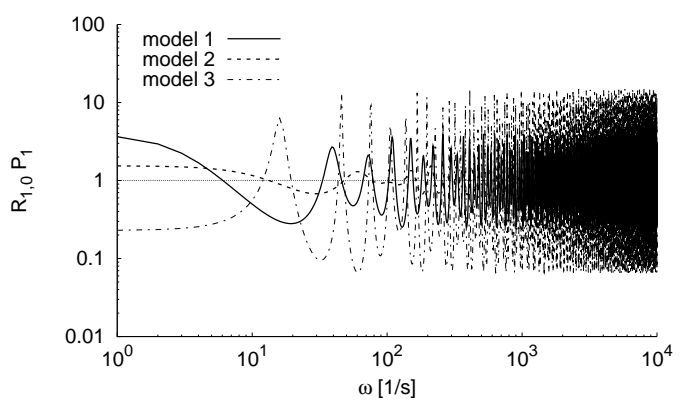

Figure 7: Samples of $P_{1}$ normalized by $1 / R_{1,0}$ for the three-layered case (Model1: $R_{1,2}=0.043, R_{2,0}=0.551, h_{1} / \beta_{1}=0.579 \mathrm{~s}, h_{2} / \beta_{2}=0.003 \mathrm{~s}$, Model2: $R_{1,2}=1.277, R_{2,0}=1.211$, $h_{1} / \beta_{1}=0.044 \mathrm{~s}, h_{2} / \beta_{2}=0.015 \mathrm{~s}$, Model3: $R_{1,2}=0.390, R_{2,0}=2.067, \quad h_{1} / \beta_{1}=0.593 \mathrm{~s}$, $\left.h_{2} / \beta_{2}=0.027 \mathrm{~s}\right)$.

random numbers within the range of $10-700 \mathrm{~m} / \mathrm{s}$ for the S-wave velocity and $1000-2000 \mathrm{~kg} / \mathrm{m}^{3}$ for the density of every layer and for Basement \#0. The total thickness of the layers is generated within the range of 1-50 $\mathrm{m}$ and then divided into layers with a random thickness.

Figure 7 shows three samples of $P_{1}(\omega)$ normalized by $1 / R_{1,0}$ for the threelayered case. In section 3 , we mentioned that the integration of average $P_{1}$ is not analytically discussed, although the calculated values, $R_{1,0} P_{1}(\omega)$, are almost distributed around 1. NED between the layers is checked in Figure 8. The integrations are also approximated by the numerical integration every $1.0 \mathrm{~s}^{-1}$ within $1.0 \mathrm{~s}^{-1} \leq \omega \leq 2.5 \times 10^{5} \mathrm{~s}^{-1}$. Every sample is on the reference line, and thus, NED is expected to be conserved through the layers even for the three- and the four-layered cases.

The simulations are applied to a ten-layered case. Five hundred sets of physical values are generated from the random numbers within the range of $10-700 \mathrm{~m} / \mathrm{s}$ for the S-wave velocity and $1000-2000 \mathrm{~kg} / \mathrm{m}^{3}$ for the density of every layer and for Basement \#0. The total thickness of the layers is generated within the range of 1-50 $\mathrm{m}$, and then divided into layers with a random thickness. NED at Layer \#1 and Basement \#0 is compared in Figure 9. The integrations are approximated by a numerical integration every $0.02 \mathrm{~s}^{-1}$ within $0.02 \mathrm{~s}^{-1} \leq \omega \leq 1.6 \times 10^{7} \mathrm{~s}^{-1}$. Almost all the samples are on the reference line, and thus, NED is expected to be conserved between Layer \#1 and Basement \#0. The depth distribution of NED normalized by that of the basement, is also shown in Figure 9. The quantities are almost constant at 1 for all the layers. Therefore, the results of the Monte Carlo 

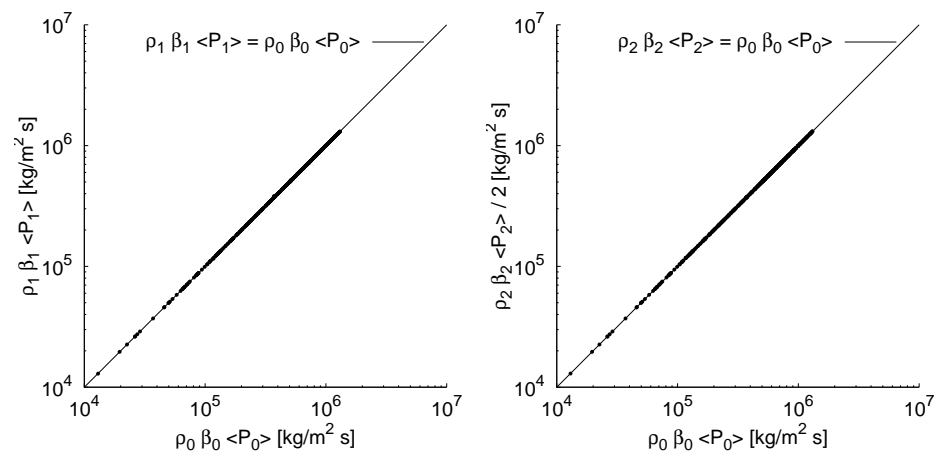

(a) three-layered case
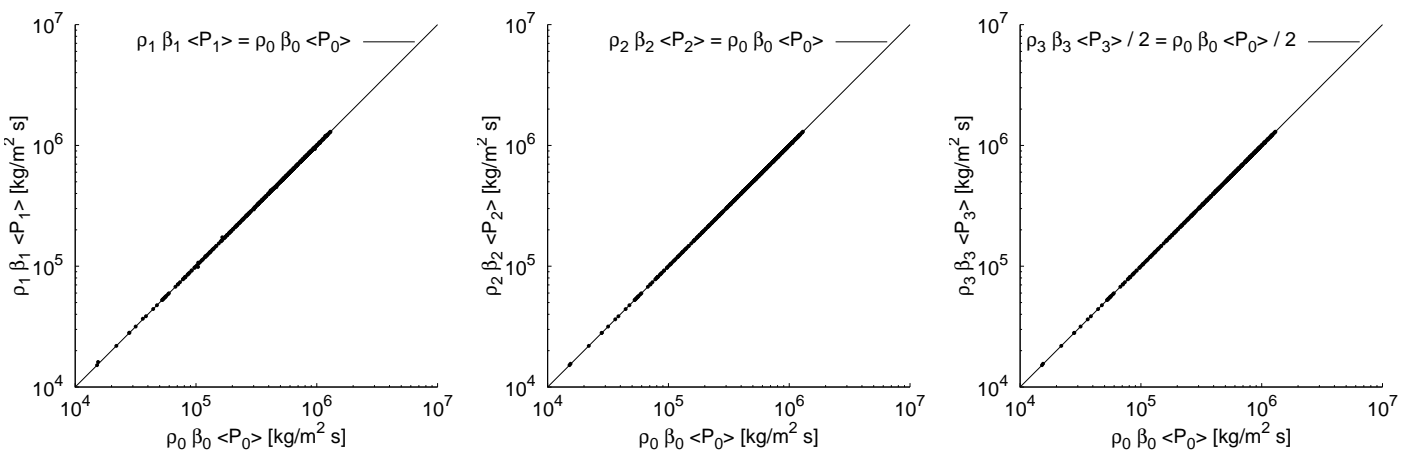

(b) four-layered case

Figure 8: Comparison of NED between Layer \#1 and Basement \#0 (top left) and between Layer \#2 and Basement \#0 (top right) for the three-layered case, and for between Layer \#1 and Basement \#0 (bottom left), between Layer \#2 and Basement \#0 (bottom middle), and between Layer \#3 and Basement \#0 (botom right) for the four-layered case. 

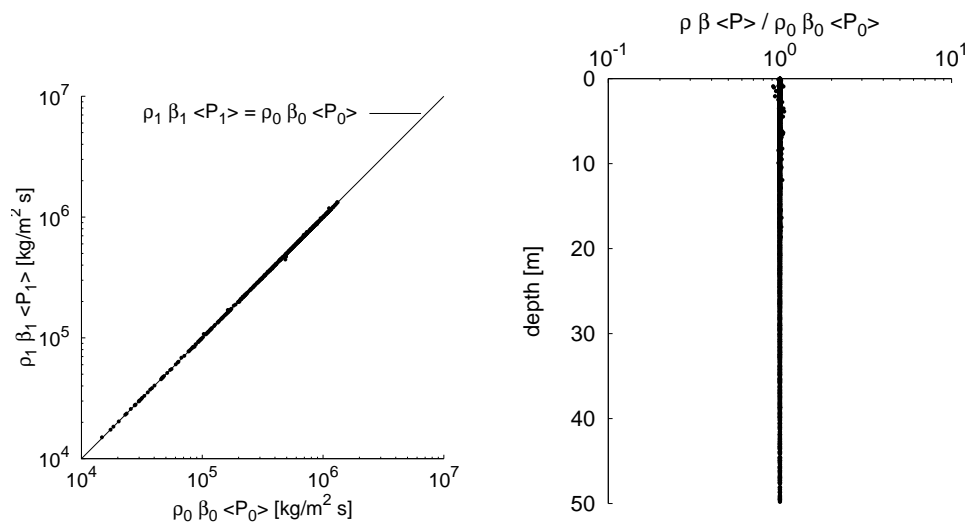

Figure 9: Comparison of NED for the ten-layered case between Layer \#1 and Basement \#0 (left), and depth distribution of NED normalized by NED at Basement \#0 (right)

simulation support the conservation of NED through the layers.

\section{Conclusions}

We introduced Normalized Energy Density that is the average power for the upgoing waves multiplied by the impedance, for a $2 \mathrm{D} \mathrm{SH}$ problem. For the two-layered case, the conservation of NED was analytically proved. For the three-, the four-, and the ten-layered cases, the conservation was verified by the Monte Carlo simulation method. The analytical proof for the general multi-layered case is still a problem; NED is supposed to be conserved through the layers.

We emphasize that NED can be evaluated from the transfer function and the impedance at the top layer. Detailed layered structures are not required for the evaluation. NED, different from the conventional definition of the energy, is conserved in structures whose detailed physical parameters have not been identified. It is anticipated that NED will be applied in the future to a wide range of studies. For example, a trade-off problem has been known to separate a contribution of source, pass and site effects by using the observed ground motions, e.g. Sato [9], Iwata and Irikura [10], Kinoshita [11], etc. A reference site is usually assumed to be no amplifications, and those at the other sites are estimated in terms of the ratio to the reference site. If the impedances on the free surface are known, NED constrains the average of site amplifications, and thus, the trade-offs might be avoided. 


\section{Acknowledgments}

We thank the two anonymous reviewers and the editor Prof. Hailan Zhang for their thorough comments. We appreciate Prof. Francisco J. SánchezSesma to have a good discussion to name the quantity Normalized Energy Density.

\section{References}

[1] A. E. H. Love, A treatise on the mathematical theory of elasticity, Dover Pubns, New York, 1927.

[2] C. Richter, An instrumental earthquake magnitude scale, Bull. Seism. Soc. Am. 25 (1935) 1-32.

[3] H. Kanamori, The energy release in great earthquakes, J. Geophys. Res. 82 (1977) 2981-2987.

[4] B. V. Kostrov, S. Das, Principles of earthquake source mechanics, Cambridge University Press, Cambridge, 1988.

[5] E. Fukuyama, Radiation energy estimated at earthquake source, Geophys. Res. Lett. 32 (2005) L13308.

[6] R. Abercrombie, A. McGarr, G. Toro, H. Kanamori, Earthquakes: radiated energy and the physics of faulting, American Geophysical Union, Washington, DC., 2006.

[7] K. Aki, P. G. Richards, Quantitativ Seismology 2nd edn, University Science Books, California, 2002.

[8] T. Kokusho, R. Motoyama, Energy dissipation in surface layer due to vertically propagating sh wave, J. Geotech. Geoenv. Eng. 128 (2002) 309-318.

[9] T. Sato, Rupture characteristics of the 1983 Nihonkai-chubu (Japan sea) earthquake as inferred from strong motion accelerograms, J. Phys. Earth. 33 (1985) 525-557.

[10] T. Iwata, K. Irikura, Source parameters of the 1983 Japan sea earthquake sequence, J. Phys. Earth. 36 (1988) 155-184. 
[11] S. Kinoshita, Frequency-dependent attenuation of shear waves in the crust of the southern Kanto area, Japan, Bull. Seism. Soc. Am. 84 (1994) 1387-1396.

\section{Appendix A. Total energy for general wave field}

Let $u_{i}$ displacement of a wave propagating to the direction $l_{i}$. The wave consists of both $\mathrm{P}$ - and S-waves.

$$
u_{i}=u_{\alpha} l_{i}+u_{\beta 1} t_{i}^{1}+u_{\beta 2} t_{i}^{2}
$$

where $u_{\alpha}$ is amplitude of $\mathrm{P}$-wave, $u_{\beta 1}$ and $u_{\beta 2}$ are the amplitudes of S-wave in $t_{i}^{1}$ and $t_{i}^{2}$ directions, which are perpendicular to $l_{i}$. When the following identities are substituted into Eq.(1),

$$
\frac{\partial u_{\alpha}}{\partial x_{i}}=-\frac{1}{\alpha} \dot{u}_{\alpha} l_{i}, \quad \frac{\partial u_{\beta}}{\partial x_{i}}=-\frac{1}{\beta} \dot{u}_{\beta} l_{i},
$$

the total energy $E$ is rewritten as follows:

$$
E=\int_{0}^{\infty} d t \int_{S}\left\{\rho \alpha \dot{u}_{\alpha}^{2} l_{i}+\rho \beta \dot{u}_{\beta}^{2} l_{i}+\rho \gamma \dot{u}_{\alpha} \dot{u}_{\beta 1} t_{i}^{1}+\rho \gamma \dot{u}_{\alpha} \dot{u}_{\beta 2} t_{i}^{2}\right\} n_{i} d S
$$

where $\gamma=\rho\left(\alpha+\beta-2 \beta^{2} / \alpha\right)$. Therefore the total energy $E$ is not equal to $E_{\alpha}+E_{\beta}$, defined by Eq.(2).

\section{Appendix B. Properties of $C_{k}$ and $D_{k}$}

From the definitions for $C_{k}$ and $D_{k}$ (Equations (27) and(28)), the following recursive formulas are obtained for $k \geq 1$.

$$
\left\{\begin{array}{l}
C_{k}=T_{11}^{k} C_{k-1}+T_{12}^{k} D_{k-1} \\
D_{k}=T_{21}^{k} C_{k-1}+T_{22}^{k} D_{k-1}
\end{array}\right.
$$

For $k=1, C_{1}$ and $D_{1}$ are explicitly represented by

$$
\left\{\begin{array}{l}
C_{1}=p_{1} \alpha_{1}+q_{1} \alpha_{1}^{*} \\
D_{1}=q_{1} \alpha_{1}+p_{1} \alpha_{1}^{*}
\end{array}\right.
$$

where $p_{k}$ and $q_{k}$ are the real variables defined by $p_{k}=\frac{1}{2}\left(1+R_{k, k+1}\right)$ and $q_{k}=\frac{1}{2}\left(1-R_{k, k+1}\right)$, and $\alpha_{k}$ is a complex variable defined by $\alpha_{k}=e^{i \omega h_{k} / \beta_{k}}$. 
$\alpha_{k}^{*}$ is the complex conjugate of $\alpha_{k}$. Thus, $D_{1}$ is proved to be the complex conjugate of $C_{1}$.

If $D_{k}$ is the complex conjugate of $C_{k}, C_{k+1}$ and $D_{k+1}$ are represented by

$$
\left\{\begin{array}{l}
C_{k+1}=p_{k+1} \alpha_{k+1} C_{k}+q_{k+1} \alpha_{k+1}^{*} D_{k}=p_{k+1} \alpha_{k+1} C_{k}+q_{k+1}\left(\alpha_{k+1} C_{k}\right)^{*} \\
D_{k+1}=q_{k+1} \alpha_{k+1} C_{k}+p_{k+1} \alpha_{k+1}^{*} D_{k}=q_{k+1} \alpha_{k+1} C_{k}+p_{k+1}\left(\alpha_{k+1} C_{k}\right)^{*}
\end{array}\right.
$$

Then, $D_{k+1}$ becomes the complex conjugate of $C_{k+1}$. Therefore, $C_{k}$ and $D_{k}$ satisfy the following relationship proved by a mathematical induction.

$$
C_{n}^{*}=D_{n}
$$

\section{Appendix C. Oblique incidence case}

Let $\delta$ to be the incident angle of the wave propagating into Basement \#0. The displacement in each layer satisfies the following wave equations:

$$
-\frac{\omega^{2}}{\beta_{k}^{2}} u_{k}=\frac{\partial^{2} u_{k}}{\partial x^{2}}+\frac{\partial^{2} u_{k}}{\partial z_{k}^{2}} .
$$

The general solution for the wave equation at Layer $\# k$ is obtained by

$$
u_{k}=A_{k} e^{i\left(k_{x} x+k_{z k} z_{k}\right)}+B_{k} e^{i\left(k_{x} x-k_{z k} z_{k}\right)},
$$

where $k_{x}$ and $k_{z k}$ are the wave number in $x$ and $z_{k}$ directions, respectively. The wave numbers are related to the S-wave velocity $\beta_{k}$ and the incident angle $\delta$, as follows:

$$
k_{x}=\frac{\omega \sin \delta}{\beta_{0}}, \quad k_{z k}=\frac{\omega}{c_{k}},
$$

where $c_{k}$ is the apparent S-wave velocity in $z_{k}$ direction defined by,

$$
c_{k} \equiv \beta_{k}\left(1-\frac{\beta_{k}^{2}}{\beta_{0}^{2}} \sin ^{2} \delta\right)^{-1 / 2} .
$$

Note that $c_{k}$ satisfies $\Re\left[c_{k}\right]>0$ and $\Im\left[c_{k}\right]>0$. When $\beta_{0}$ is greater than $\beta_{k} \sin \delta, c_{k}$ is a real number.

From the boundary condition at the interface between Layer \# $(k+1)$ and $\# k$, the amplitudes for Layer \# $(k+1)$ are represented by those for Layer $\# k$ as

$$
\left(\begin{array}{c}
A_{k+1} \\
B_{k+1}
\end{array}\right)=\left[\begin{array}{ll}
\frac{1}{2}\left(1+\tilde{R}_{k, k+1}\right) e^{i \omega h_{k} / c_{k}}, & \frac{1}{2}\left(1-\tilde{R}_{k, k+1}\right) e^{-i \omega h_{k} / c_{k}} \\
\frac{1}{2}\left(1-\tilde{R}_{k, k+1}\right) e^{i \omega h_{k} / c_{k}}, & \frac{1}{2}\left(1+\tilde{R}_{k, k+1}\right) e^{-i \omega h_{k} / c_{k}}
\end{array}\right]\left(\begin{array}{c}
A_{k} \\
B_{k}
\end{array}\right),
$$


where $\tilde{R}_{k, k+1}$ is defined by

$$
\tilde{R}_{k, k+1} \equiv \frac{\rho_{k} \beta_{k}^{2} / c_{k}}{\rho_{k+1} \beta_{k+1}^{2} / c_{k+1}} .
$$

The above system of equations are the same form with Eq.(21). When $c_{k}$ is a real number in each layer, $\tilde{R}$ becomes a real number, and $e^{i \omega h_{k} / c_{k}}$ and $e^{-i \omega h_{k} / c_{k}}$ are complex numbers with the absolute values of 1 . In the case, $\left\langle P_{k}\right\rangle$, defined by $\left\langle\left|A_{k} / A_{0}\right|^{2}\right\rangle$, is the same with Eq.(33) if impedance and Swave velocity are exchanged to $\rho_{k} \beta_{k}^{2} / c_{k}$ and $c_{k}$, respectively. Therefore, the oblique incident case is identical to the problems of vertical incidence with the impedance of $\rho_{k} \beta_{k}^{2} / c_{k}$ and S-wave velocity of $c_{k}$ if $\beta_{0}>\beta_{k} \sin \delta$ is satisfied in each layer. 